\title{
A job ladder model with stochastic employment opportunities
}

\author{
JAKE BRADLEY \\ School of Economics, University of Nottingham and IZA \\ Axel GotTfries \\ School of Economics, University of Edinburgh
}

\begin{abstract}
We set up a model with on-the-job search in which firms infrequently post vacancies for which workers occasionally apply. The model nests the standard job ladder and stock-flow models as special cases, while remaining analytically tractable and easy to estimate from standard panel data sets. The parameters from a structurally estimated model on US data are significantly different from either the restrictions imposed by a stock-flow or job ladder model. Imposing these restrictions significantly understates the search option associated with employment and are, unlike our model, inconsistent with recent survey evidence and declining job finding rates and starting wage with duration of unemployment, both of which are present in the data.
\end{abstract}

KeYwords. On-the-job search, wage dispersion, wage posting, stock-flow.

JEL CLASSIFICATION. J31, J64.

\section{INTRODUCTION}

Due to their effectiveness in replicating unemployment, worker turnover, and wage dynamics, on-the-job search models are extensively used to evaluate labor market policies. ${ }^{1}$ Embedded in the standard job ladder model is a single friction, modeled as a Poisson process, that prevents the reallocation of workers into more productive jobs. In this paper, we extend the standard job ladder model by adding an additional state variable

Jake Bradley: jake.bradley@nottingham.ac.uk

Axel Gottfries: axel .gottfries@gmail .com

First version: April 21, 2017. We would like to thank Vasco Carvalho, Mike Elsby, Per Krusell, Hannes Malmberg, Kurt Mitman, Fabien Postel-Vinay, Pontus Rendahl, and Kjetil Storesletten for advice and comments. We would also like to acknowledge participants at the BGSE Summer Forum (SAM) 2017, University of Cambridge, Conference on Markets with Search Frictions, EALE 2017, Essex Search and Matching workshop, IIES, Nordic Summer Symposium in Macroeconomics 2017, University of Nottingham, University of Oxford, SAEe and SED 2017 for useful feedback and suggestions. Gottfries gratefully acknowledge financial support from the Tom Hedelius Foundation and the UK Economic and Social Research Council (ESRC), Award reference ES/L009633/1. Both authors gratefully acknowledge financial support by the Keynes Fund under the project JHON. All errors are our own.

${ }^{1}$ Recent evaluations include enforcement policy on informal firms (Meghir, Narita, and Robin (2015)), public sector wage and employment policy (Bradley, Postel-Vinay, and Turon (2017)) and tax policy (Sleet and Yazici (2017)).

(c) 2021 The Authors. Licensed under the Creative Commons Attribution-NonCommercial License 4.0. Available at http://qeconomics.org. https://doi.org/10.3982/QE1394 
on workers, their employment opportunities. ${ }^{2}$ These suitable vacancies are posted infrequently by firms and applications to these are made intermittently by workers. As special cases, our model nests the standard job ladder model and a version of the stockflow model. After estimating the model, we find that the restrictions implied by both the standard job ladder model and the stock flow model leads to a significant underestimate of the search option associated with employment. Furthermore, the model is consistent with survey evidence on work search behavior and generates a declining job finding rate with the duration of unemployment and substantial wage losses following displacement, which increase in the duration of unemployment, all of which the standard job ladder model fails to generate.

In the model, only some job openings are suitable for a given worker. ${ }^{3}$ This set of job opportunities is treated as a latent variable that follows a stochastic process. Similar to McCall (1970), firms create suitable job openings at some Poisson rate and at some Poisson rate, a firm will stop looking for workers. In addition, as in Stigler (1962), workers infrequently send out multiple applications. The worker subsequently accepts the best offer, if it is better than her current job. We allow for different rates by employment status to capture any differential search behavior. To match the data, we allow firms to differ in productivity and workers have an individual skill component. We close the model by assuming that firms post wage schedules in worker productivity, prior to meeting the worker. ${ }^{4}$ In deciding on the optimal wage, a firm trades off the higher chance of hiring a worker and the longer expected duration of the match against the higher wage cost. The resulting model nests the workhorse empirical labor models of Burdett and Mortensen (1998), and models of stock-flow matching, pioneered by Coles and Smith (1998), as special cases. Like Burdett and Mortensen (1998), the model has an analytical closedform solution and is well identified and empirically tractable, allowing us to estimate all of the parameters using a panel data set on wage and employment dynamics.

We estimate the model using a two-step procedure assuming that the labor market is segmented by the workers' level of education. In the first step, the parameters governing workers' search behavior are identified by the flows between labor markets states and the duration dependence of the transition rate from unemployment to employment. These moments are calculated from the Current Population Survey (CPS). In the second step, the parameters governing worker and firm productivity distributions are identified

\footnotetext{
${ }^{2}$ A suitable vacancy or a labor market opportunity, in the context of our model, is defined as at the point of application, a job that a worker is: (i) aware of; and (ii) suitable for. To describe these suitable vacancies from a worker's perspective, we will use the terms opportunities and prospects interchangeably.

${ }^{3}$ There is a number of papers emphasizing differences, (e.g., skill, location) between available vacancies and the unemployed, creating thin markets, and thereby resulting in the simultaneous coexistence of unemployed workers and vacancies; see, for example, Lucas and Prescott (1974), Coles and Smith (1998), Shimer (2007), Alvarez and Shimer (2011), and Carrillo-Tudela and Visschers (2013). In our model, the stock of job opportunities can be interpreted as the local conditions for the worker and a number of the unemployed have no prospects and are therefore "mismatch" unemployed. Furthermore, the mismatch and stock-flow family of models generate similar employment dynamics as our model and are also able to generate the declining job finding rate with the duration of unemployment.

${ }^{4}$ The main results of the paper are unchanged if the worker and the firm instead bargain over the wage after the match has been formed. The implications of alternative assumptions on the nature of wage setting are discussed in Section 2.7.
} 
from the distribution of average wages across workers as well as the overall distribution of wages. Wage moments are computed using the Survey of Income and Program Participation (SIPP). The estimated model matches the transition rates from employment to unemployment and out of the labor force and the declining job finding rate with the duration of unemployment. In the context of the model, the decline of the job finding rate with the duration of unemployment is informative of the importance of worker's employment prospects. The job finding rate declines with the duration of unemployment because the newly unemployed have, on average, more prospects than the long-term unemployed. In the job ladder model, all unemployed are the same, that is, unemployment is a single state, which implies that the model is unable to match the falling job finding rate with the duration of unemployment. ${ }^{5}$

The estimated parameters imply that unemployed workers become aware of fewer suitable vacancies compared to the employed. Unemployed workers send out applications more often: twice a month compared to less than twice a year for their employed counterparts. This is consistent with Faberman, Mueller, Şahin, and Topa (2016) who document that the unemployed send out a much larger number of applications but the number of contacts are similar. The relation between the empirical observation of Faberman et al. (2016) and our estimated search process is explored in Section 3.7. In our model, unemployment is both due to the infrequent applications made by workers as well as the workers lack of suitable opportunities. If all workers were to have some prospects, that is, the limit as their arrival rate goes to infinity, the unemployment rate would fall by a bit more than a half. In contrast, in the benchmark model of Burdett and Mortensen (1998), unemployment disappears as the arrival rate of job contacts increase. The relative importance of frequency of applications versus availability of prospects differs starkly by employment state suggesting that the one friction representation of the labor market might be particularly poor.

Hornstein, Krusell, and Violante (2011) suggested that a particularly suitable metric for assessing the option value of search is the ratio of the mean to the minimum wage, hereafter the mean-min ratio $(\mathrm{Mm})$. In order to generate a $\mathrm{Mm}$ ratio of close to two, our estimation of the Burdett and Mortensen (1998) model requires a large negative flow benefit associated with unemployment whereas our estimated baseline model matches the same frictional wage distribution with replacement ratios in the order of $25-50 \%$. This difference can be decomposed into two channels. First, employed workers receive more job offers when they apply which shows up as if they were to sample wages from a distribution that stochastically dominates that of their unemployed counterparts (consistent with recent evidence from Faberman et al. (2016)). Second, consistent with the data, after losing their job, a worker recently made unemployed will, on average, find a job more quickly than the long-term unemployed. Interpreting the data through the lens of the Burdett and Mortensen (1998) model thus overestimates the foregone search

\footnotetext{
${ }^{5}$ Belot, Kircher, and Muller (2019) ran an experiment whereby job seekers are exposed to a greater number of vacancies. The treated had an increased number of interviews and particularly so for the long-term unemployed. This result is broadly consistent with our estimated model, which suggests that an important driver of unemployment is a lack of labor market opportunities rather than the frequency at which unemployed workers apply.
} 
option, and hence underestimates the flow benefit associated with unemployment. This estimated replacement ratio is consequential for the ability of models to generate cyclical fluctuations in the unemployment rate. ${ }^{6}$

In a search model without OJS or stochastic match quality, there is no wage loss following displacement as the average outstanding wage is equal to the average starting wage. In a job ladder model, on the other hand, workers gradually select into better paying jobs. The average employed worker will thus receive a higher wage than the average worker coming from unemployment. Thus, a displaced worker will, on average, experience a wage loss, but these losses do not increase with the duration of unemployment. General human capital depreciation in unemployment generates wages falling with the duration of unemployment and thereby persistent losses. Researchers studying earnings losses using job ladder models have often incorporated falling general human capital in unemployment as an important ingredient. ${ }^{7}$

However, falling general human capital would entail falling reservation wages with the duration of unemployment which is inconsistent with recent evidence from Krueger and Mueller (2016). In addition, since all unemployed search in the same market, the standard assumption of log linear production (and benefits) generates a constant job finding rate with the duration of unemployment. If instead the matching set were to decrease, the continuously falling human capital would imply the same for the job finding rate, which is inconsistent with the empirical observation that the job finding rate falls quickly in the first 3 months but is broadly constant thereafter. Our model, in addition to featuring a positive selection into better jobs, also features an additional state variable-employment prospects. When the model is estimated, we find that the newly unemployed do, on average, have more prospects than the long-term unemployed. This implies that the job finding rate and starting wages fall with the duration of unemployment as, via dynamic selection, workers with more prospects exit and those without prospects remain. Our model thus jointly fits: a falling job finding rate with the duration of unemployment and a large wage loss following displacement that is increasing in the duration of unemployment.

Similar to our paper, there is a number of search papers with multiple meetings and applications. However, the key friction in these models differs from our own. A large number of papers have modeled a thick market where workers make multiple applications and are able to direct their search (Albrecht, Gautier, and Vroman (2006), Kircher

\footnotetext{
${ }^{6}$ Shimer (2005) illustrated how the Diamond-Mortensen-Pissarides model with replacement ratios in line with unemployment insurance generosity does not generate the empirically observed variations seen in market tightness (level of vacancies divided by unemployment) given the low variation in labor productivity over the business cycle. If the flow benefit of unemployment is high, then the profit will be low and the model is able to generate a sufficient amplification to productivity shocks (Hagedorn and Manovskii (2008)). Elsby and Michaels (2013) considered a model in which firms exhibit decreasing returns to scale allowing replacement rates to be low compared to average productivity but still high in comparison to the marginal productivity. In our paper, we find that the flow value of unemployment is indeed high compared to the average wage in line with Shimer (2005) and Elsby and Michaels (2013).

${ }^{7}$ See, for example, Krolikowski (2017), Jarosch (2015), and Burdett, Carrillo-Tudela, and Coles (2020) for recent papers studying earnings losses.
} 
and Galenianos (2009), Kircher (2009), Wolthoff (2017)). ${ }^{8}$ Closer to our paper are recent papers where workers are unable to direct their search but meet multiple firms (Elliott (2014), Wolthoff (2014), Gautier and Holzner (2017)). In all of these models, either the number of applications a worker can make is exogenous, or each application carries an additional cost. Workers are ex ante homogeneous in their market conditions and ex post heterogeneous in their position in a network. Our model takes a complementary approach where instead workers are ex ante heterogeneous in the thickness of their individual markets, and the number of potential opportunities follows a stochastic process.

Following Hornstein, Krusell, and Violante (2011), a number of recent papers have examined the ability of search models to generate sufficient frictional wage dispersion. Either there has to be an additional effect that offsets the foregone search option, or it must be that the search option is not measured correctly. For example, if human capital depreciates quickly in unemployment, then that can motivate workers to take low paid jobs (Ortego-Marti (2016)). Such an explanation would entail reservation wages falling quickly with the duration of unemployment which contradicts recent survey evidence (Krueger and Mueller (2016)). Within a sequential auctions framework, like in PostelVinay and Robin (2002) and Cahuc, Postel-Vinay, and Robin (2006), the bargaining position of the worker increases when the worker takes a new job. These models can then generate more wage dispersion via this foot in the door effect (Papp (2013)). A foot in the door effect is also present in Carrillo-Tudela (2009) where there is no search option in unemployment. Faberman et al. (2016) is the closest to this paper. They consider a job ladder model with exogenously different wage offer distributions for employed and unemployed workers. When a worker is employed, the lower arrival rate of job offers is partly offset by a better offer distribution. In our model, the offer distribution is a time varying object but, on average, the distribution faced by the employed stochastically dominates the distribution facing the unemployed. Our paper can thus be seen as a microfoundation for the two exogenous offer distributions in Faberman et al. (2016).

Outline. The rest of this paper is structured as follows. In Section 2, we set up the model and provide the analytical solution. In Section 3, we present the estimation of the model and the quantitative results. Section 4 concludes the paper.

\section{Model}

\subsection{The environment}

Time is continuous and the labor market is populated by risk-neutral workers and firms. Workers leave the labor force at a Poisson rate $\mu$ and are replaced by a doppelgänger in unemployment. ${ }^{9}$ Workers are ex ante heterogeneous in their productivity $x$, distributed with the cumulative distribution $\Gamma_{x}(\cdot)$ and ex post vary in their employment state $s \in\{u, e\}$, their employment opportunities $j$, and if employed, their wage w. Firms

\footnotetext{
${ }^{8}$ In these models, the worker faces two problems: a portfolio problem in deciding which jobs to apply for; as well as the optimum number of applications to send out.

${ }^{9}$ In this setting, the parameter $\mu$ acts in an isomorphic manner to a discount factor.
} 
are infinitely lived and are heterogeneous in their productivity $y$. The cumulative distribution of productivity amongst vacancies is given by $\Gamma_{y}(\cdot)$. Both worker and firm productivity distributions are primitives of the model. We will use $F$ to refer to a firm's rank in the productivity distribution. The total output of a match is the product of worker and firm types, $x y(F)$. In unemployment, workers earn a flow income proportional to their productivity type, $b x$. Jobs become unprofitable at an exogenous rate $\delta$, which results in the worker entering the pool of unemployed. Finally, we do not allow workers to quit, other than to move to a new job. ${ }^{10}$

The frictions. A worker is aware of some number of vacancies. However, only a subset of these constitute genuine opportunities, that is, a vacancy that the worker is suitable for, and thus would receive an offer were they to apply. A worker amasses job opportunities according to a Poisson rate $\lambda_{s}$ that differs by the employment state $s$ of the worker. This Poisson rate represents the posting of a vacancy that the worker is both suitable for and aware of. For this reason, the Poisson rate may differ by employment state (consistent with evidence from Faberman et al. (2016)). For example, employed workers could learn about opportunities in other firms through referral networks at their places of work. We assume that once a worker has learned of an opportunity it is not forgotten. The rate at which these opportunities disappear comes instead from the firm ceasing to hire workers, which happens at Poisson rate $v$.

We assume that the worker applies to their stock of opportunities $j$ at a Poisson rate $\gamma_{s}$. One interpretation is that a worker incurs a fixed cost in time when applying to jobs. The process described is similar to one in which free time arrives at a Poisson rate. The frequency at which workers have available time to look for jobs will likely vary with their employment state. Our parameter estimates suggest that, relative to the employed, the unemployed apply far more frequently. We argue that this is also borne out by the data. Krueger and Mueller (2012) found that in the U.S. while the unemployed engage in active job search more often than the employed, $20 \%$ spend some time in a given day looking for work compared to $0.6 \%$ of the employed. Conditional on looking, both groups spend a large and comparable time searching, approximately 160 minutes for the unemployed and 100 minutes for the employed.

A finite value for the application rate $\gamma_{s}$ implies that workers match with the stock intermittently. Some workers will have no opportunities and will wait for the flow to increase their stock of opportunities, which is analogous to a worker waiting to match with the flow in a standard discrete time stock-flow matching model. When the worker matches with the stock of vacancies, they choose the most appropriate prospect.

In the model, after an offer has been turned down, be it for an alternative job prospect or staying with a current employer, a worker cannot subsequently return to that offer. We think that this captures a realistic feature of the labor market and is analogous to the standard assumption in the job ladder models that previous jobs cannot be

\footnotetext{
${ }^{10}$ Consider an environment in which workers are aware of detailed information regarding their prospects (e.g., the latent number of opportunities in their set and the wages associated with them). If $\gamma_{u}>\gamma_{e}$, for those earning a low wage and with good prospects, the value of unemployment to the worker could potentially exceed the value of continued employment. In the estimated model, this affects a negligible number of workers and would affect no one if unemployment insurance is not paid to voluntary quits or if there is a sufficiently high minimum wage.
} 
recalled. ${ }^{11}$ An alternative modeling choice would be to assume that workers can hold on to rejected offers until the firm stops looking for workers. This would somewhat complicate the exposition without being quantitatively important as vacancies and, therefore, prospects have a short shelf life. We calibrate the rate of expiry $v$ later based on the duration of a vacancy and find that job opportunities last approximately 1 month. This is an order of magnitude larger than the rate at which employed workers, for example, switch jobs and would likely therefore have little effect on our results. In Appendix A.1.1 (see Supplementary Material, Bradley and Gottfries (2021)), we describe the flow equations for the distribution of job opportunities, $j \in \mathbb{N}_{+}$.

Each employment prospect arrives with a wage that is set optimally by profit maximizing firms. Firms can post wages conditional on worker type $x$. In equilibrium, the wage that a firm of productivity rank $F$ pays a worker of productivity $x$ is denoted by the wage function $w(x, F)$.

\subsection{Worker problem}

An individual's utility is given by the present expected discounted value of their future income stream. It turns out to be convenient to write all value functions in terms of a worker's employment status, opportunity stock and, if employed, their wage. We wish to remain agnostic on exactly what is contained in the information set of the worker regarding their opportunities. One interpretation, is that a worker is aware of a set of vacancies but not for which of those they are suitable. They only become aware of the number of true opportunities and their associated wage offers after they apply to the stock. Importantly, since the meetings are governed by a Poisson process, the equilibrium function is the same even if workers had access to more information. The value function for an unemployed worker of type $x$ with $j$ offers in hand, is given by (1):

$$
\begin{aligned}
\mu U(x, j)= & b x+\gamma_{u} \int_{0}^{1}(\max \{W(x, w(x, F), 0), U(x, 0)\}-U(x, j)) d F^{j} \\
& +\lambda_{u}(U(x, j+1)-U(x, j))+v j(U(x, j-1)-U(x, j)),
\end{aligned}
$$

where $F \in[0,1]$ is the rank of wages from the job offer sampling distribution. ${ }^{12}$ The value function of an unemployed worker, discounted by the rate at which she leaves the market, is the sum of the flow value of unemployment $b x$. The search option of the worker is given by the rate at which she accesses the market, $\gamma_{u}$, multiplied by the expected returns to matching with the stock. $W(\cdot)$ is the value of employment and defined in (2). Notice that when an unemployed worker takes up a job offer, she begins her employment spell with no opportunities. This is because the worker has rejected all other offers and we assume there is no recall of offers previously turned down. While in unemploy-

\footnotetext{
${ }^{11}$ Recall of previous jobs has recently been explored by Fujita and Moscarini (2017) and Carillo-Tudela and Smith (2016) in a Mortensen and Pissarides (1994) and a sequential auctions model of the labor market, respectively.

${ }^{12}$ Notice that this corresponds perfectly with a firm's rank in the productivity distribution. This is because, as will be seen, firms pay all workers they employ the same piece rate wage and this is a monotonically increasing function in a firm's productivity type.
} 
ment, the number of employment opportunities of a worker follows a stochastic process with suitable jobs arriving at a rate $\lambda_{u}$, and losing a given opportunity at a rate $v$.

Given the underlying latent variable, the value function associated with employment can be written as the sum of the contracted flow wage $\mathrm{w}$, the option value of the $j$ opportunities in the worker's stock, the option value of the stochastic process that governs the evolution of $j$ and the option value of becoming unemployed, which occurs with probability $\delta$,

$$
\begin{aligned}
\mu W(x, \mathrm{w}, j)= & \left.\mathrm{w}+\gamma_{e} \int_{0}^{1}(\max \{W(x, w(x, \tilde{F}), 0), W(x, \mathrm{w}, 0)\}-W(x, \mathrm{w}, j))\right) d F^{j} \\
& +\lambda_{e}(W(x, \mathrm{w}, j+1)-W(x, \mathrm{w}, j))+v j(W(x, \mathrm{w}, j-1)-W(x, \mathrm{w}, j)) \\
& +\delta(U(x, j)-W(x, \mathrm{w}, j)) .
\end{aligned}
$$

As in a standard sequential search model, a worker's decision is whether to accept or reject a given offer. ${ }^{13}$ Once matching with the stock, a worker has potentially more than one offer to contend with. Since the wage lasts forever and all jobs are otherwise homogeneous, a worker will always prefer the highest wage job available to them, be it in the stock of opportunities or the job they are currently employed in. An unemployed worker accepts a wage if it yields a higher present value than continuing in unemployment. Since firms post wages optimally, no firm would post a wage less than this value (assuming it is lower than their productivity) and, therefore, we are solving for the infimum of the wage support, $\phi(x)=w(x, 0)$. This is found by solving the equality

$$
U(x, 0)=W(x, \phi(x), 0) .
$$

Solving the reservation wage is slightly more difficult than usual due to the evolution of the additional state variable-the number of employment opportunities. Appendix A.2 explains how one can compute the value functions and derives an expression for $\phi(x)$.

\subsection{Steady-state distribution of match quality}

In order to solve for the distribution of wages and outstanding matches, we proceed in two steps. First, we define the probability generating function $\Sigma_{s}$ for each employment state $s$ as

$$
\Sigma_{s}(F)=\sum_{j=0}^{\infty} p_{s}(j) F^{j},
$$

where $p_{s}(j)$ is a probability mass function that gives the probability that a worker in state $s$ has exactly $j$ employment opportunities. The function $\Sigma_{s}(F)$ evaluates the probability

\footnotetext{
${ }^{13}$ One could imagine a more sophisticated set of strategies depending on what the worker is aware of (e.g., the number of vacancies in the stock, her job tenure or the wages of individual job opportunities). In such an environment, employed workers would under some conditions optimally quit to unemployment. However, this is beyond the scope of this paper.
} 
that when a random worker in state $s$ matches with the stock, she has no vacancy above the $\operatorname{rank} F$. The function $\Sigma_{S}(F)$ has the steady-state solution

$$
\begin{aligned}
\Sigma_{e}(F)= & \frac{1}{1-F} \int_{F}^{1} \exp \left[-\lambda_{e} / \delta(\tilde{F}-F)\right]\left(\frac{1-\tilde{F}}{1-F}\right)^{\frac{\gamma_{e}+\mu}{\delta}} \frac{\gamma_{e}+\mu+\delta}{\delta} d \tilde{F}, \\
\Sigma_{u}(0)= & \frac{\int_{0}^{1} \exp \left[-\lambda_{u} / \delta \tilde{F}\right](1-\tilde{F})^{\frac{\gamma_{u}-\delta}{\delta}}\left[\frac{\gamma_{u} \Sigma_{e}(\tilde{F})}{\delta}\right] d \tilde{F}}{1-\int_{0}^{1} \exp \left[-\lambda_{u} / \delta \tilde{F}\right](1-\tilde{F})^{\frac{\gamma_{u}-\delta}{\delta}}\left[\frac{\gamma_{u}}{\delta}\left(1-\Sigma_{e}(\tilde{F})\right)\right] d \tilde{F}}, \\
\Sigma_{u}(F)= & \frac{1}{1-F} \int_{F}^{1} \exp \left[-\lambda_{u} / \delta(\tilde{F}-F)\right]\left(\frac{1-\tilde{F}}{1-F}\right)^{\frac{\gamma_{u}-\delta}{\delta}} \\
& \times\left[\frac{\gamma_{u} \Sigma_{u}(0)}{\delta}+\frac{(\delta+\mu)(1-u) / u \Sigma_{e}(\tilde{F})}{\delta}\right] d \tilde{F} .
\end{aligned}
$$

The derivation of this function is in Appendix A.1.2. The rate of inflow into unemployment from employment is given by $\delta+\mu$. Similarly, the rate of outflow from unemployment is given by $\gamma_{u}\left(1-p_{u}(0)\right)$. In steady-state, the inflow is equal to the outflow which, using the definition of $\Sigma_{u}(F)$, gives an expression for the unemployment rate

$$
u=\frac{\delta+\mu}{\delta+\mu+\gamma_{u}\left(1-\Sigma_{u}(0)\right)} .
$$

The total unemployment rate contains both the friction at which workers qualify for jobs and the frequency at which they apply. In a hypothetical case in which, $\lambda_{u} \rightarrow \infty$, all workers have some employment prospects, that is, $p_{u}(0)=0$, and then the unemployment rate will purely be due to workers not sending out enough applications and given by

$$
\tilde{u}=\frac{\delta+\mu}{\delta+\mu+\gamma_{u}} .
$$

Comparing the true unemployment rate with the one in which all workers have employment opportunities reveals the relative importance of the two frictions for the unemployment rate. Using the function $\Sigma_{s}(F)$, we can further solve for the distribution of outstanding matches $G(F)$. Note that the inflow of matches below $F$ is $\gamma_{u} \sum_{j=1}^{\infty} F^{j} p_{u}(j)$, that is, the probability that an unemployed worker matches with an offer less than $F$. Similarly, the outflow of matches below $F$ is the exogenous separation $\delta+\mu$ plus the endogenous quit of $\gamma_{e}\left(1-\sum_{j=0}^{\infty} F^{j} p_{e}(j)\right)$. In steady state, the inflow must equal the out flow, which gives

$$
(1-u) G(F)\left(\delta+\mu+\gamma_{e}\left(1-\sum_{j=0}^{\infty} F^{j} p_{e}(j)\right)\right)=u \gamma_{u}\left(\gamma_{u} \sum_{j=1}^{\infty} F^{j} p_{u}(j)\right)
$$


Using the definition of $\Sigma_{s}$, we get

$$
G(F)=\frac{u \gamma_{u}\left(\Sigma_{u}(F)-\Sigma_{u}(0)\right)}{(1-u)\left(\delta+\mu+\gamma_{e}\left(1-\Sigma_{e}(F)\right)\right)} .
$$

The associated density function and its derivative are given in Appendix A.1.3.

\subsection{Firm problem}

The firm commits to a wage schedule in worker productivity at the time of vacancy creation. The firm then sets the wage to optimally trade off the increased retention and hiring with the increased cost associated with a higher wage. The expected profits per vacancy for a firm with match quality rank $F$ posting a wage $w$ are made up by three terms: the probability that a worker is hired; the expected duration; and the markup. Combining these gives the expression for the expected profits at the time of vacancy creation

$$
\Pi(x, w, F)=\operatorname{Pr}(\text { hire } \mid x, w) \mathrm{E}(\text { duration } \mid x, w)(y(F) x-w) .
$$

Since a firm's size is proportional to $\operatorname{Pr}($ hire $\mid x, w) \mathrm{E}($ duration $\mid x, w)$, this is equivalent to Burdett and Mortensen (1998) where the firm maximizes steady state profits.

Hiring. Search is random, a firm posting a vacancy can either meet an employed or unemployed worker. For the worker to accept the offer, the wage has to be higher than any other offer the worker holds and, if employed, her current wage. Absent of market thickness dynamics, that is, the Burdett and Mortensen (1998) model, workers match instantaneously, which means that the offer is always the best amongst the new job offers. The wage is acceptable if it is above the current wage or the reservation wage for the unemployed. In contrast, without OJS, all contacted workers are unmatched but potentially receive more than one offer as in Burdett and Judd (1983). Either of these mechanisms will generate equilibrium wage dispersion. Our model combines both aspects as there is both search on the job and workers match with the stock. The probability that a worker is hired conditional on meeting can then be calculated using

$$
\begin{aligned}
\operatorname{Pr}(\text { hire } \mid w \geq \phi)= & \operatorname{Pr}(\text { meet an unemployed worker }) \operatorname{Pr}(\text { unemployed worker accepts } \mid w) \\
& +\operatorname{Pr}(\text { meet an employed worker }) \operatorname{Pr}(\text { employed worker accepts } \mid w) .
\end{aligned}
$$

The probability that a vacancy meets an unemployed worker, conditional on a meeting, is the flow rate of meetings with unemployed workers divided by the total flow rate of all meetings. The flow rate of meetings for unemployed workers comprises the product of three terms: the rate at which the worker engages in active search; the stock of unemployed; and the expected number of opportunities. The expected number of job opportunities is given by $\sum_{j=1}^{\infty} j p_{s}(j)$.

The probability that the worker accepts the offer, conditional on meeting with the vacancy, can be broken up into two parts. The probability that the offer is better than her current offer ( 1 for the unemployed and $G(w)$ for the employed) times the probability that the offer is the highest among all offers the worker has received. The probability that 
an offer with wage rank $F$ is the highest offer among all offers for the worker in state $s$ is the probability that the vacancy meets a worker with $j$ offers $\frac{j p_{s}(j)}{\sum_{j=1}^{\infty} j p_{s}(j)}$, multiplied by the probability that the offer is higher than the $j-1$ alternative offers $\left(F^{j-1}\right)$. This gives

$$
\frac{\sum_{j=1}^{\infty} j p_{s}(j) F^{j-1}}{\sum_{j=1}^{\infty} j p_{s}(j)}
$$

Combining the expressions above and using the definition of $\Sigma_{s}$, we get

$$
\begin{aligned}
\operatorname{Pr}(\text { hire } \mid w(F) \geq \phi)= & \frac{\gamma_{u} u \sum_{j=1}^{\infty} j p_{u}(j)}{\gamma_{u} u \sum_{j=1}^{\infty} j p_{u}(j)+\gamma_{e}(1-u) \sum_{j=1}^{\infty} j p_{e}(j)} \frac{\sum_{j=1}^{\infty} j p u(j) F^{j-1}}{\sum_{j=1}^{\infty} j p_{u}(j)} \\
& +\frac{\gamma_{e}(1-u) \sum_{j=1}^{\infty} j p_{e}(j)}{\gamma_{u} u \sum_{j=1}^{\infty} j p_{u}(j)+\gamma_{e}(1-u) \sum_{j=1}^{\infty} j p_{e}(j)}(1-u) G(F) \frac{\sum_{j=1}^{\infty} j p_{e}(j) F^{j-1}}{\sum_{j=1}^{\infty} j p_{e}(j)} \\
= & \frac{\left(\gamma_{u} u \Sigma_{u}^{\prime}(F)+\gamma_{e}(1-u) G(F) \Sigma_{e}^{\prime}(F)\right)}{\left(\gamma_{u} u \Sigma_{u}^{\prime}(1)+\gamma_{e}(1-u) \Sigma_{e}^{\prime}(1)\right)} .
\end{aligned}
$$

Duration of a job. Unlike in Burdett and Mortensen (1998), the duration of a job is not exponentially distributed. Instead, the quit rate is a time varying object. It turns out that even though the leaving rate is not constant, the average leaving rate is a sufficient statistic for the expected duration at the time of hiring due to Little's law. We can calculate the expected duration at the time of hiring. The average rate at which a worker working in a firm of productivity rank $F$ leaves the job is given by $\delta+\mu+\gamma_{e}\left(1-\Sigma_{e}(F)\right)$. The average duration in a job $F$ is therefore just $1 /\left(\delta+\mu+\gamma_{e}\left(1-\Sigma_{e}(F)\right)\right)$.

\subsection{Equilibrium}

An equilibrium in this economy is characterized by: the value functions $\{U(x, j)$, $W(x, w, j), \Pi(x, w, F)\}$ given by (1), (2), and (8), respectively; a wage function $w(x, F)$ that maximizes the present value of profits (8); and a reservation wage $\phi(x)$ solving equation (3). In this environment, a firm's optimal strategy will be to post piece rate contracts in worker type, as in Barlevy (2008), since all flow income scales with workers' productivity $x$. With a slight abuse of notation, we can express the wage function as $w(x, F)=x w(F)$ and the value functions as $U(x, j)=x U(j)$ and $W(x, w(x, F), j)=$ 
$x W(w(F), j)$. The piece rate wage $w(F)$ solves the firm problem, such that (8) is maximized, and the worker is indifferent between the lowest wage and unemployment, both absent of opportunities $W(x, \phi(x), 0)=U(x, 0)$ with $\phi(x)=x w(0)$.

\subsection{Identification}

In this subsection, we discuss the identification the transition parameters $\left\{\mu, \delta, \lambda_{u} \lambda_{e}\right.$, $\left.\gamma_{u}, \gamma_{e}\right\}$. Intuitively, the rate of application $\gamma_{u}$ and $\gamma_{e}$ determines the job finding rate in unemployment and the job-to-job transition rate, respectively. The values of $\lambda_{u}$ and $\lambda_{e}$ instead determine how the job finding rate changes with duration in unemployment and employment, respectively. Appendix A.3 provides a proof that these transition parameters of our model are identified relying only on transition moments (thus, justifying the use of a two-step procedure). Although the exact moments discussed in the Appendix A.3 are not practically implemented in the estimation, we use similar moments for the purpose of estimation. The aim of our estimation is to minimize a criterion defined as a distance between simulated and empirically observable moments. Identification of worker and firm productivity is discussed in Section 3.3 and relies on the specific parametric assumptions.

\subsection{Discussion}

Relation to existing models. Notice that a number of commonly used search models are nested in our framework. First, in the absence of dynamic market thickness, that is, as $\gamma_{s} \rightarrow \infty \forall s \in\{e, u\}$. The model converges to the standard job ladder model of Burdett and Mortensen (1998). In the limit, as workers continuously apply, a worker has to immediately decide whether to accept or reject a given offer, and hence, there are never multiple offers to contemplate simultaneously. Second, with no on-the-job search, $\gamma_{e}=0$, and with applications made continuously by the unemployed, $\gamma_{u} \rightarrow \infty$, the model nests a version of the stock-flow matching function in continuous time; see Coles and Smith (1998). In this case, after separating from a firm, the worker will immediately match with the stock of their available opportunities. If the stock is nonempty, the worker will directly transition to a new job, whereas if the stock is empty, the worker has to wait for the inflow of new opportunities. Lastly, if $\gamma_{e}=0$, and the unemployed infrequently apply for jobs, $\gamma_{u} \in(0, \infty)$, then the model shares the feature of stock-flow matching but with search frictions. This case corresponds closely to a dynamic version of the Burdett and Judd (1983) model. We estimate the baseline model as well as the model without dynamics of market thickness (NDT), and without on-the-job search (NOJS).

Discussion of wages. In Burdett and Mortensen (1998) and Bontemps, Robin, and den Berg (2000), like in our model, the firm trades off the hiring and retention of workers against a higher wage cost. The first-order condition for the logarithm of expected profits gives a differential equation for the optimal wage. Defining $h(F)=\partial \log \operatorname{Pr}($ hire $) / \partial F$ and $r(F)=\partial \log E$ (duration) $/ \partial F$ and $w^{m}(F)=\int_{0}^{F} m(F)(y(F)-w(F)) d F$ where, $m \in\{h, r\}$, we can decompose the wage $w(F)$ into three terms, the wage increase from the reten- 
tion motive $\left(w^{r}(F)\right)$ and the hiring motive $\left(w^{h}(F)\right)$ and the wage that satisfies the participation constraint for the worker $w(0)$,

$$
w(F)=w^{r}(F)+w^{h}(F)+w(0) .
$$

In the Burdett and Mortensen (1998) model, the motive to pay for retention and hiring is

$$
r(F)=h(F)=\frac{\lambda_{e}}{\delta+\mu+\lambda_{e}(1-F)} .
$$

In our model, the incentives are

$$
\begin{aligned}
h(F) & =\frac{\left(\gamma_{u} u \Sigma_{u}^{\prime \prime}(F)+\gamma_{e}(1-u) G^{\prime}(F) \Sigma_{e}^{\prime}(F)+\gamma_{e}(1-u) G(F) \Sigma_{e}^{\prime \prime}(F)\right)}{\left(\gamma_{u} u \Sigma_{u}^{\prime}(F)+\gamma_{e}(1-u) G(F) \Sigma_{e}^{\prime}(F)\right)}, \\
r(F) & =\frac{\gamma_{e} \Sigma_{e}^{\prime}(F)}{\left(\delta+\mu+\gamma_{e}\left(1-\Sigma_{e}(F)\right)\right)} .
\end{aligned}
$$

In Appendix A.6, Figure A.3, we show the fraction of the wage that is paid due to the incentive to retention workers. The results suggest that the hiring motive is quantitatively more important, but relatively less so higher up in the upper support of the wage distribution.

We can now compare how the firm's incentive to increase wages depends on the estimated parameters. The percentage increase in the firm size with firm type, $\ell^{\prime}(F) / \ell(F)$, is just $\ell^{\prime}(F) / \ell(F)=G^{\prime \prime}(F) / G^{\prime}(F)=h(F)+r(F)$ and we can rewrite the first-order condition as

$$
\Pi_{w}(w, F)=\frac{\ell^{\prime}(F)}{\ell(F)}(y(F)-w(F))-w^{\prime}(F)=0 .
$$

We refer to $G^{\prime \prime}(F) / G^{\prime}(F)$ as the degree of competition. If $G^{\prime \prime}(F) / G^{\prime}(F)$ is low, then the firm size is unresponsive to the wage rank and there is thus little reason to increase the pay. The degree of competition, using our formula, can be written as

$$
\frac{\ell^{\prime}(F)}{\ell(F)}=\frac{\Sigma_{u}^{\prime \prime}(F)+\gamma_{e} \frac{\Sigma_{u}(F)-\Sigma_{u}(0)}{\delta+\mu+\gamma_{e}\left(1-\Sigma_{e}(F)\right)} \Sigma_{e}^{\prime \prime}(F)}{\Sigma_{u}^{\prime}(F)+\gamma_{e} \frac{\Sigma_{u}(F)-\Sigma_{u}(0)}{\delta+\mu+\gamma_{e}\left(1-\Sigma_{e}(F)\right)} \Sigma_{e}^{\prime}(F)}+2 \gamma_{e} \frac{\Sigma_{e}^{\prime}(F)}{\delta+\mu+\gamma_{e}\left(1-\Sigma_{e}(F)\right)},
$$

whereas the competition term for the Burdett and Mortensen (1998) model is

$$
\frac{\ell^{\prime}(F)}{\ell(F)}=\frac{2 \lambda_{e}}{\delta+\mu+\lambda_{e}(1-F)} .
$$

Note that $\Sigma_{s}(F)$ is a convex function. When the firm considers the hiring margin in the Burdett and Mortensen (1998) model, it need only consider the probability that the worker is working at a lower paying firm. In our setup, the firm also needs to consider 
the probability that the worker has a better offer in hand. The competition in our model therefore increases more as we move to the tail of the distribution.

The Burdett and Mortensen (1998) model fails to generate much wage competition in the upper tail of the distribution. Our model includes a further competition term via a Burdett and Judd (1983) mechanism. In order to show the difference, we plot expression (9) to calculate the competition for different firm types for Burdett and Mortensen (1998) and our model. Figure A.2, in the Appendix A.5, reveals, that for all skill groups, high productive firms exhibit a stronger wage competition in our model as compared to Burdett and Mortensen (1998). The intuition for this is that since some workers have many offers, it is relatively more likely that the highest offer is in the upper part of the distribution. The competition at the lower type firm is, on the other hand, similar in our model and that in the Burdett and Mortensen (1998) model. The introduction of thin markets into the Burdett and Mortensen (1998) model thus shifts competition from the lower to the upper part of the distribution, thereby increasing the dispersion of wages for a given primitive firm productivity distribution.

Alternative wage setting mechanisms. There are numerous alternative approaches to wage setting in models with on-the-job search. The contribution of our paper is in the modeling of the frictions governing worker reallocation which could, in principle, be incorporated with any wage setting protocol. We therefore opt for a simple wage setting mechanism, which is common in the literature whilst also doing justice to the data. It should also be noted that the nature of wage setting is hard to identify in a short panel without relying on strong functional form assumptions.

We follow Burdett and Mortensen (1998), an environment in which firms post and commit to wages prior to meeting the worker and subsequently do not match outside offers. This approach is taken for a number of practical reasons. First, it is used frequently in the literature. Second, many other wage setting mechanisms will result in only minor differences in the estimated parameters. In fact, if the wage setting results in efficient transitions, ${ }^{14}$ only the parameters of the production functions and value of home production will differ depending on the determination of wages. ${ }^{15}$ Third, the equilibrium with this wage setting is robust to timing assumptions and the exact specification of the information set of workers and firms which is not necessarily true for some of the other commonly used alternatives in the literature. ${ }^{16}$

\footnotetext{
${ }^{14}$ That is a worker, in equilibrium, accepts a job offer if and only if the productive is higher than that of their current employer.

${ }^{15}$ Optimal back-loaded wage contracts in the spirit of Burdett and Coles (2003) and Stevens (2004) are notable examples of a wage setting protocol in which efficient transitions does not (necessarily) hold; see Burdett and Coles (2010).

${ }^{16}$ Consider a protocol in which outside offers are matched, as in Postel-Vinay and Robin (2002). If offers arrive sequentially, the value associated with unemployment is the discounted flow benefit of unemployment. However, if job offers arrive simultaneously, firms would compete with each other resulting in a higher value associated with unemployment. Similarly, if wages are renegotiated infrequently without offer matching as in Gottfries (2019). The incentive to pay a higher wage to retain the worker would depend on the number of prospects $j$, and thereby on the exact information set of the worker.
} 


\section{Estimation}

Our estimation will focus on estimating the model presented in the previous sections. In addition, we estimate the special cases of no dynamics of market thickness (NDT) and without OJS (NOJS).

\subsection{Data}

The data used in estimation are taken from the Current Population Survey (CPS) and the Survey of Income and Program Participation (SIPP). Moments relating to labor mobility are taken from the CPS, due to its larger cross-sectional component. Wage moments are taken from the SIPP. The sample is stratified according to observable skill level into three distinct strata, consistent across data source. They are: the college educated; those whose highest academic achievement is a high school diploma; and those who have not completed their high school education. ${ }^{17}$ We restrict attention to male workers aged between 25 and 45 in order to best mitigate issues associated with early retirement and nonparticipation. In order to give the alternative specifications ample chance of matching the level of frictional wage dispersion, we trim the bottom $10 \%$ of the wage distribution. ${ }^{18}$ Moreover, we restrict the attention to the relatively short and stable period between the years 1996 and 1999, inclusive. As will be seen, key parameters will be identified from labor mobility by duration and we do not want cohort effects to play any role. In Supplementary Appendix S.2, we plot the separation and job finding rates by age. Separation rates exhibit a clear downward trend and the pattern of the finding rate is less clear. Since our model assumes a constant separation rate, we choose a window where this seems to be a fair approximation of the data. Specific details regarding sample selection are provided in the Supplementary Appendix.

The identification relies on employment dynamics and the cross-sectional wage distribution. Table 1 reports moments on hourly earnings and the number of hours worked per week for each stratum. These are computed by dividing the self-reported weekly earnings by self-reported hours worked per week. Since in the estimation, wage data are taken from the SIPP and employment dynamics from the CPS, it is reassuring that the data in both look quantitatively similar. The two data sets are broadly consistent. Respondents in the SIPP are, on average higher skilled and work a greater number of hours. There are large systematic differences in hourly earnings across skills. These differences are the motivation for stratification. Comparing hourly earnings across strata seems sensible as there is little cross strata variation in hours. Finally, it is worth bearing in mind that the medium-skilled, those with a high school diploma but without a college degree, account for about half of the labor force.

Table 2 presents employment dynamics, estimated from a three-state Markov process. All moments presented in Table 2 will be matched in the estimation to come. In

\footnotetext{
${ }^{17}$ The particularities of the stratification are detailed in the Supplementary Appendix (Bradley and Gottfries (2021)).

${ }^{18} \mathrm{We}$ do this so not to overstate the mean-min ratio as survey data are susceptible to measurement error (see Bound, Brown, and Mathiowetz (2001)) particularly at the extremum of the wage distribution.
} 
TABle 1. Descriptive statistics.

\begin{tabular}{lcccc}
\hline & All & Low Skill & Medium Skill & High Skill \\
\hline $\begin{array}{l}\text { Proportions: } \\
\text { CPS }\end{array}$ & $100 \%$ & $11 \%$ & $52 \%$ & \\
SIPP & $100 \%$ & $9 \%$ & $51 \%$ & $40 \%$ \\
Mean Earnings (\$/hour): & & & & \\
CPS & 15.8 & 9.8 & 13.9 & 20.2 \\
SIPP & 15.7 & 9.6 & 13.4 & 19.9 \\
Mean Weekly Hours Worked: & & & & \\
CPS & 43.1 & 41.4 & 42.5 & 44.2 \\
SIPP & 44.9 & 43.5 & 44.4 & 45.9 \\
\hline
\end{tabular}

Note: Data come from the Monthly Outgoing Rotation Group (MORG) of the CPS and the on-seam months of the SIPP, months that do not rely on recall. Moments are based on male workers aged between 25 and 45 between 1996 and 1999 , inclusive. The CPS data contains 163,093 observations and the SIPP 85,972. All moments presented here and elsewhere are computed using the appropriate sampling weights.

TABLE 2. Transition rates of the employed.

\begin{tabular}{lcccc}
\hline & All & Low Skill & Medium Skill & High Skill \\
\hline probability in $t+1$ employed worker is: & & & & \\
Inactive & 0.010 & 0.020 & 0.010 & 0.006 \\
Unemployed & 0.012 & 0.026 & 0.013 & 0.006 \\
Employed by different employer & 0.026 & 0.033 & 0.026 & 0.023 \\
Number employed in sample & 584,920 & 58,415 & 304,319 & 222,186 \\
\hline
\end{tabular}

Note: The transition rates are monthly. Data are taken from the CPS and relate to 25-45-year-old males between 1996 and 1999, inclusive.

addition, the job finding rate of the unemployed is targeted by duration of the unemployment spell. The largest differences in employment dynamics across strata are the frequency to which workers exit employment, rather than the frequency to which they are hired from unemployment; see Figure 1. The higher the skill of a worker, the longer the expected employment duration with lower exit probabilities to inactivity, unemployment, or an alternative employer.

\subsection{Parameterization}

The set of parameters to be estimated is given by the vector $\theta$,

$$
\theta=\left(\mu, \delta, v, \lambda_{u}, \lambda_{e}, \gamma_{u}, \gamma_{e}, b, \Gamma_{x}(x), \Gamma_{y}(y)\right)^{\prime} .
$$

We assume the labor market is segmented by observable skill, as well as gender and the age cutoff described in Section 3.1. The vector $\theta$ is estimated for each skill and the economy at large. Notice that (10) contains the entire distributions of $\Gamma_{x}(x)$ and $\Gamma_{y}(y)$. We make further parametric assumptions on the primitive initial distribution of worker and firm types. We assume that the distributions follow transformed log-normals. With 
a slight abuse of notation, we define a worker's rank in the distribution as $F_{x}$ and recall that a firm's rank is $F$. $\Phi$ denotes a standard normal distribution. For firm's we include location and shape parameters, given by $\mu_{y}$ and $\sigma_{y}$ and for worker's a shape parameter, $\sigma_{y}$ :

$$
x\left(F_{x}\right)=\exp \left[\sigma_{x} \Phi^{-1}\left(F_{x}\right)\right] \text { and, } \quad y(F)=\exp \left(\mu_{y}\right)+\exp \left[\sigma_{y} \Phi^{-1}(F)\right] .
$$

The rate at which workers lose employment opportunities, $v$, is the only parameter not directly estimated and calibrated to match the mean duration of a vacancy. Vacancy duration is estimated using the "Conference Board Help Wanted Online Data Series" (HWOL) using a simple relation between the stock and the flow. Details of exactly how this parameter is calibrated are provided in Appendix A.4. It should be noted that these data do not cover our estimation window nor can we look at the vacancy duration by skill requirement of the job opening. The implied value of the mean duration $(1 / v)$ is approximately 1 month. ${ }^{19}$

After these assumptions, equation (10) can be reduced to the following vector of scalars. The focus of the rest of this section is the estimation of the vector $\theta$,

$$
\theta=\left(\mu, \delta, \lambda_{u}, \lambda_{e}, \gamma_{u}, \gamma_{e}, b, \sigma_{x}, \mu_{y}, \sigma_{y}\right)^{\prime} .
$$

\subsection{Estimation protocol}

The model is estimated by indirect inference in two steps. In a first step, employment transitions are matched, based on CPS data. The second step matches auxiliary wage moments computed from the SIPP to uncover the underlying primitive productivity distributions of workers and firms and the value of home production. To estimate the models of no OJS search and no dynamic market thickness, we use an identical first step. In order to match the same degree of frictional wage dispersion, we compute the distribution predicted by our baseline model and target this directly. To do this, we use a more flexible distribution to guarantee a satisfactory fit. ${ }^{20}$ Thus, for the two alternative specifications, we do not estimate the distribution of worker types.

Transition rates. The first step matches aggregate job-to-job, employment to unemployment, and the rate at which workers leave the labor market. Finally, the job finding rate of the unemployed are computed by duration. The job finding rate of the unemployed is computed as a monthly probability at a weekly frequency for the first 12 weeks of their unemployment spell. To avoid sparsity, we then compute the monthly probability at a monthly frequency for the following 9 months. This allows us to target precisely the steep initial decline in the hazard rate and avoids time windows later in the spell where no transitions are observed. We thus match 24 moments, three aggregate rates, and 21 job finding rates by duration of unemployment spell, which we weight

\footnotetext{
${ }^{19}$ Notice, we assume the duration of a vacancy is orthogonal to a worker's skill. With more detailed microdata regarding the skill requirements of a job, theoretically this parameter could also be conditioned on worker skill. However, as we argue in Appendix S.4, quantitative results do not appear to depend on the specific calibration of $v$.

${ }^{20}$ We include an additional scale parameter such that $y(F)=\exp \left(\mu_{y}\right)+\zeta_{y} \exp \left[\sigma_{y} \Phi^{-1}(F)\right]$. Notice that for $\zeta_{y}=1$ it becomes identical to the distribution of firm types in the baseline.
} 
by the precision to which they are estimated in the data. This step is matched varying $\theta^{t}=\left(\mu, \delta, \lambda_{u}, \lambda_{e}, \gamma_{u}, \gamma_{e}\right)$ and can be done independently of all other parameters. Formally, $\theta^{t}$ is the solution to the following:

$$
\hat{\theta}^{t}:=\arg \max _{\theta^{t} \in \Theta^{t}}\left(m^{t}\left(\theta^{t}\right)-m^{t}\right)^{\prime} \hat{V}^{-1}\left(m^{t}\left(\theta^{t}\right)-m^{t}\right),
$$

where $m^{t}\left(\theta_{t}\right)$ and $m^{t}$ are the 25 targeted moments, from the model and data, respectively, and $\hat{V}$ is the diagonal of the variance-covariance matrix of $m^{t}$. It is worth pointing out that the estimation attributes all duration dependence in the data through our model's specific channel. There are of course likely many other sources that we could potentially account for. We have ignored mechanisms in order to make the results easier to interpret. The other extreme case of no duration dependence corresponds to the restriction imposed under the model of no dynamic thickness.

Wage distributions. This step estimates the value of home production and worker and firm productivity parameters, $\theta^{p}:=\left(b, \sigma_{x}, \mu_{y}, \sigma_{y}\right)$. We simulate data generated from our model as in the SIPP. That is, we simulate a monthly panel with the same number of individuals, over the same time frame, with the same rate of attrition. Since we only rely on the seam of the SIPP, where wages are not based on recall, we treat the simulated data in the same way. In order to distinguish between the relative contribution of worker and firm productivity, we match each (1st to 99th) percentile of the mean wage of a worker over our time horizon. Further, we match the same percentiles of the overall wage distribution including the infimum of the support. This leaves a total of $199 \mathrm{empir}-$ ical moments to fit, which we denote by the vector $m^{p}$. To review this, $m^{p}$ consists of the 100 quantiles of the wage support $\left\{w_{q}\right\}_{q=0, \ldots 99}$ and the 99 quantiles of the mean worker wage $\left\{\bar{w}_{q}\right\}_{q=1, \ldots 99}$.

Unlike step one, we do not have any analytical expressions for our moments, but instead rely on Monte Carlo simulations. We simulate the model $M$ times and take the mean of each model predicted moment condition, given by $M^{-1} \sum_{i=1}^{M} m_{i}^{p}\left(\theta^{p}\right)$. Further, since the empirical distribution of wages contains excess mass at round numbers and there is simulation error, a bootstrapped weighting matrix does not seem appropriate. Instead, we implement a two-step GMM estimation in which the first step estimates the weighting matrix, $W(\theta)^{-1}$. In the first step, take an initial guess at $W(\theta)^{-1}$ as the identity matrix and estimate the model. We then simulate $m_{i}^{p}\left(\theta^{p}\right) 500$ times and compute a variance-covariance matrix as our estimate of $W(\theta)$. The second step estimates $\theta^{p}$ as the solution to

$$
\hat{\theta^{p}}:=\arg \max _{\theta^{p} \in \Theta^{p}}\left(\frac{\sum_{i=1}^{M} m_{i}^{p}\left(\theta^{p} ; \theta^{t}\right)}{M}-m^{p}\right)^{\prime} \hat{W}(\theta)^{-1}\left(\frac{\sum_{i=1}^{M} m_{i}^{p}\left(\theta^{p} ; \theta^{t}\right)}{M}-m^{p}\right) .
$$

Practicalities. In the second step of the estimation, we resimulate our model 500 times, $M=500$. In order to isolate differences across specifications, we first estimate 
our model. Then, in the two special cases, we fix the distribution of worker productivity to be identical to our model. In this estimation of the two special cases (no market thickness and no OJS), we include an additional scale parameter of the firm productivity distribution $\zeta_{y}$ to ensure a satisfactory fit. In all three specifications the first step is identical, but for the two nested cases, the second step matches the distribution of wages simulated from our baseline model. That is, target 99 percentiles of the $G(w)$ distribution predicted by the baseline model as above, using the identity matrix as a weighting matrix.

Identification. As with targeting the transition rates, this step is overidentified. Unlike the first step, however, identification relies on heuristic argument. Changing any of the five elements of the vector $\theta^{p}$ will change all 199 targeted moments. However, certain parameters speak directly to certain moments in the data. The level of wages is driven primarily by the level of firm productivity, in particular the location parameter $\mu_{y}$. The dispersion of a worker's mean wage by the dispersion in worker type $\sigma_{y}$. Consider the case of workers being homogeneous in productivity. Given a sufficiently long time horizon, in which workers are observed in many different jobs, one would expect to see little or no dispersion in worker's mean wage. Since all wage heterogeneity is governed by the firm in which they are matched and not any systematic differences across workers. The dispersion of firm types explains any residual dispersion in the overall wage distribution: the so-called level of frictional wage dispersion. Finally, given all other elements in $\theta^{p}$, the value of home production $b$ pins down the lowest observed wage.

\subsection{Results}

Running the multistep estimation procedure as described yields the parameter estimates presented in Table 3. The first rows presents the transition parameters followed by the replacement ratio. The final cell of Table 3 presents the parameters from the underlying distributions of worker and firm productivity. Bootstrapped standard errors are given in parentheses and all parameters are statistically significant to any conventional significance level.

Fit. Figure 1 shows the probability that an unemployed worker moves to employment, by the duration of her unemployment spell. The horizontal dotted line represents that predicted with dynamic thickness (NDT). The declining solid line is our baseline model and the crosses and dashed line are the targeted moments from the data. We omit the special case of no OJS as it is indistinguishable from the baseline. All models do almost exactly match the aggregate transition rates. Appendix S.3 displays the fit of the wage distributions. Figure S.2 shows the fit of the overall wage distribution for the baseline model. Figure S.3 displays the fit of the distribution of mean worker wages. Both distributions are skewed to the right for all worker types.

Transition parameters. Inspecting the transition parameters, the upper cell of Table 3 , it is immediately apparent that each model has a very different interpretation of the functioning of the labor market. First, across all skill groups, in the model without market thickness (NDT) $\lambda_{u}>\lambda_{e}$, which implies that workers are exposed to a greater number of job offers in unemployment than in employment. The higher rate at which 


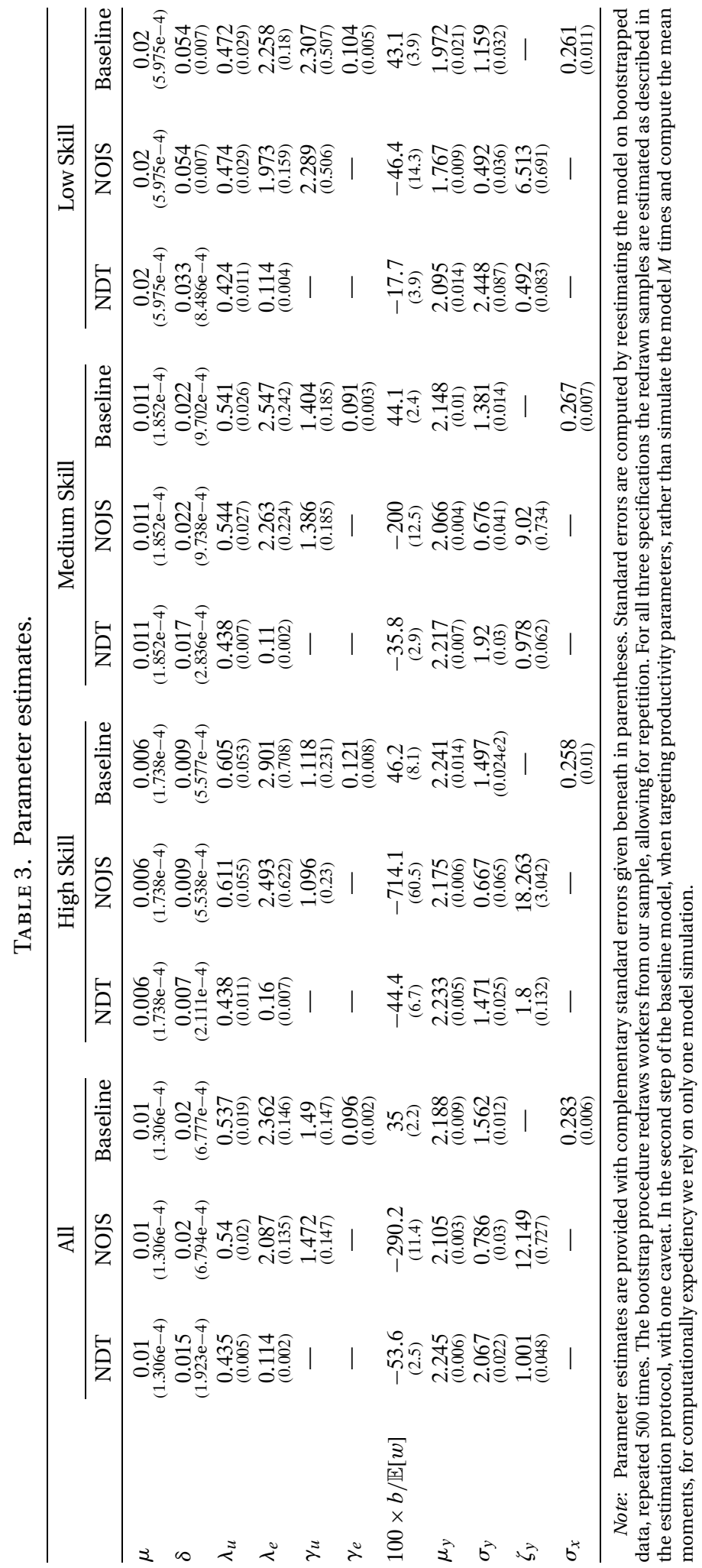


High Skill

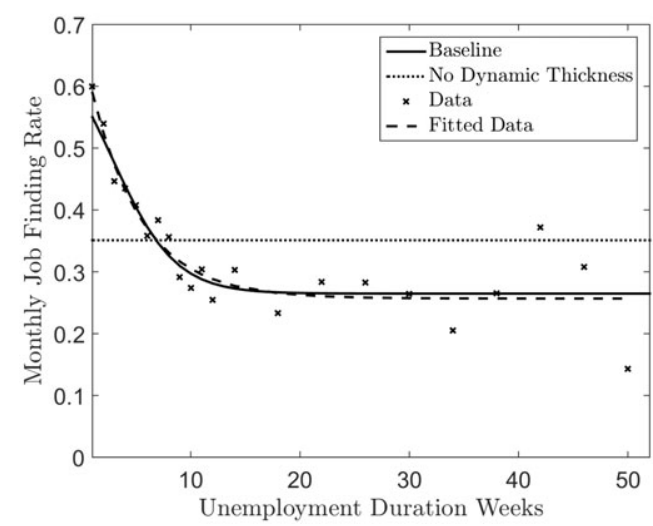

Low Skill

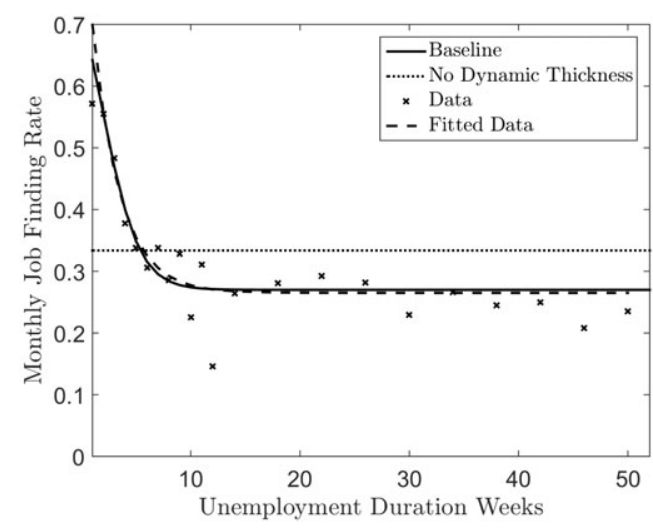

Medium Skill

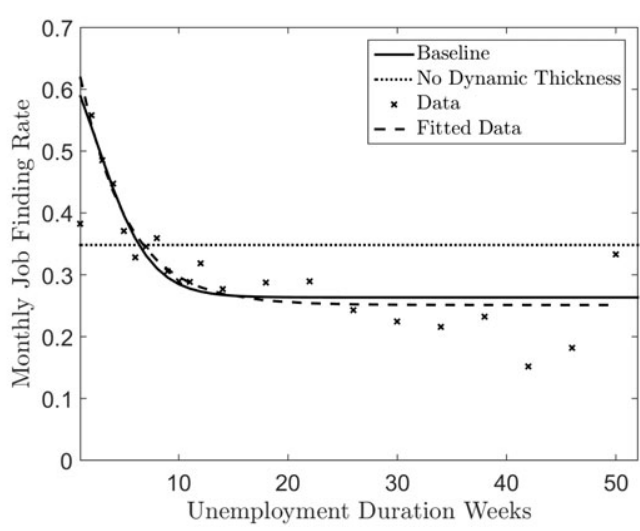

All

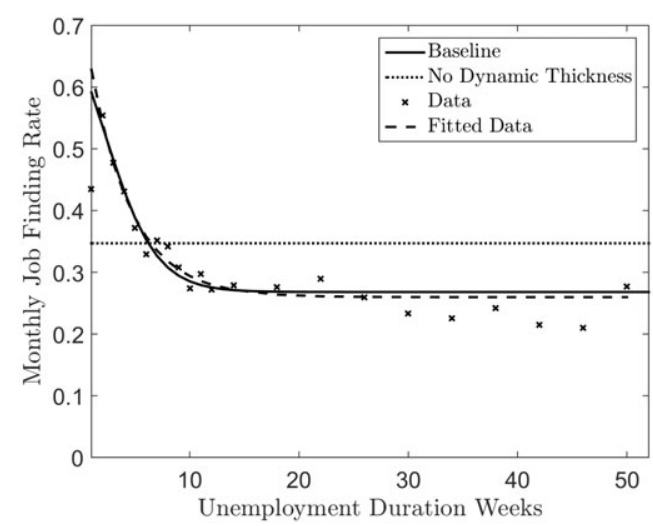

Figure 1. Job finding rate by duration of unemployment. Note: The empirical monthly job finding rate, represented by an ' $x$ ', is computed at a weekly frequency for the first 12 weeks and a monthly frequency thereafter. The dashed black line represents the fitted relationship $\beta_{1}+\beta_{2} \exp \left(-\beta_{3} \tau\right)$, where $\tau$ is unemployment duration (in weeks) and the $\beta$ s are estimated by nonlinear least squares according to the same criterion as is used in the estimation.

the unemployed find new jobs is rationalized by a higher contact rate in NDT. Finally, across all skill groups, the job destruction rate $\delta$ is higher in the model with stochastic market thickness. In that version, the newly unemployed find jobs more quickly than they would in the NDT framework and, consequently, more workers lose their jobs and find employment within a month. ${ }^{21}$

Duration dependence decomposition. The duration dependence can be decomposed into two parts. First, there is a dynamic selection whereby those with more prospects are more likely to leave unemployment. Second, a worker's set of opportunities evolve

\footnotetext{
${ }^{21} \mathrm{By}$ the same logic, the baseline model also generates declining $E \rightarrow U$ monthly transitions with the duration of employment. The number of opportunities is on average increasing with the duration of an employment spell, which increases the probability of returning to employment within the month.
} 
High Skill

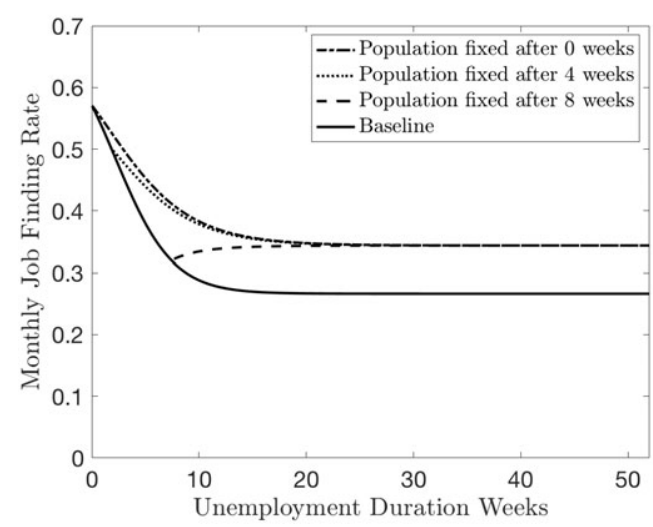

Low Skill

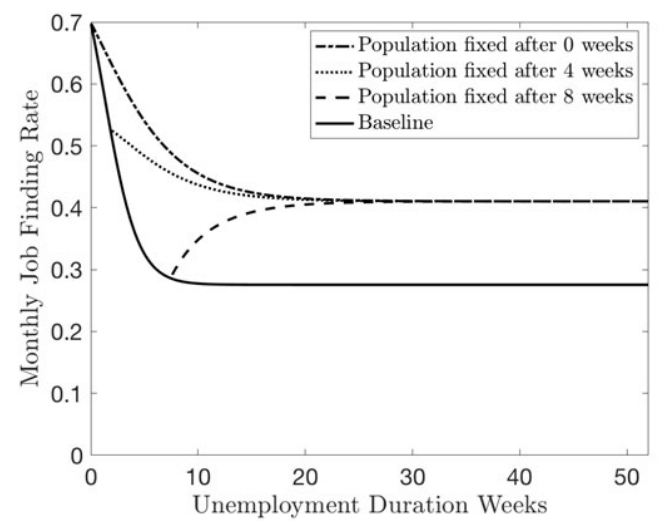

Medium Skill

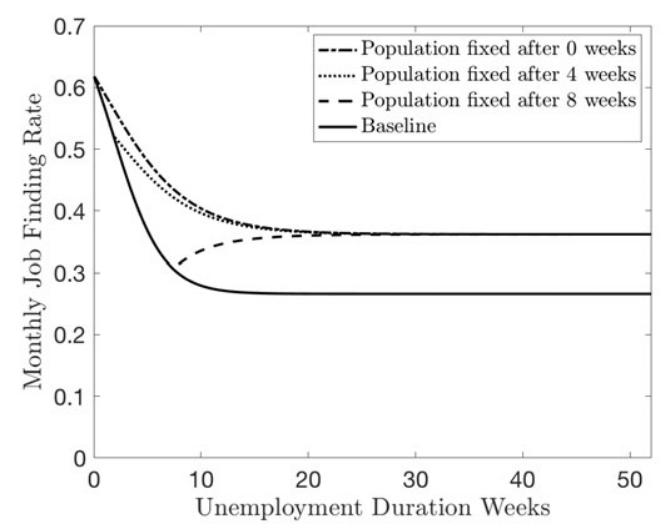

All

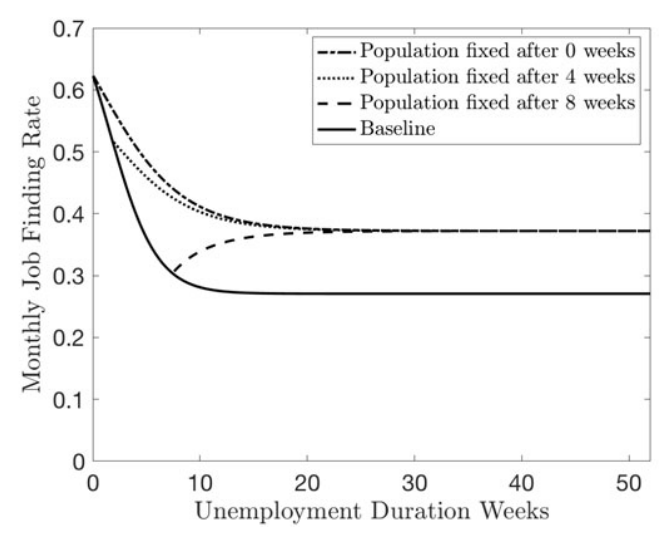

Figure 2. Duration dependence decomposition. Note: The black solid line represents the monthly job finding rate predicted by the model by unemployment duration. The dashed lines are intended to compute the duration dependence absent of dynamic selection. This is done by computing the job finding rate assuming the pool of unemployment is fixed-workers remaining in unemployment after finding a job.

stochastically over their unemployment spell. The latter can be isolated by computing the job finding rate for workers assuming that those that find a job stay in the pool of unemployed. $^{22}$ The exercise is inspired by the procedure of Alvarez, Borovickova, and Shimer (2019) who decompose duration in a stopping time model. Figure 2 performs this exercise fixing the population of unemployed after 0,4 and 8 weeks of unemployment. As discussed, the job finding rate converges to an ergodic rate. Ignoring dynamic selection, by fixing the pool of unemployment, the true duration dependence converges to a higher level.

The selection effect always acts to decrease the job finding rate-as only those with positive opportunities leave the pool of unemployment. However, the true duration de-

\footnotetext{
${ }^{22}$ We thank an anonymous referee for this suggestion.
} 
TABLE 4. Unemployment decomposition.

\begin{tabular}{lcccc}
\hline Unemployment Rate & All & High Skill & Medium Skill & Low Skill \\
\hline$\tilde{u}$ & $2.0 \%$ & $1.4 \%$ & $2.3 \%$ & $3.1 \%$ \\
$u$ & $4.4 \%$ & $2.6 \%$ & $4.9 \%$ & $8.5 \%$ \\
\hline
\end{tabular}

Note: This table computes and compares $u$ and $\tilde{u}$ as defined in equations (4) and (5).

pendence depends on the distribution of opportunities. Workers who have been unemployed for shorter lengths of time have on average more opportunities but are on average losing them as time lapses. We therefore see a monotonic decline in the hazard rate when we fix the pool for shorter periods. However, after longer periods, workers are on average accumulating opportunities which explains why when the population of the unemployed is fixed after 8 weeks we see an increase in the job finding rate. In fact, for long a duration the two channels perfectly offset, which explains why the job finding rate converges to a constant level. We interpret share of the decline of the job finding rate, which is captured when the population is held fixed as the relative importance of true duration dependence. Using this metric and the entire sample, true duration dependence explains approximately $70 \%$ of the fall in the job finding rate. We attribute the final $30 \%$ to dynamic selection.

Unemployment decomposition. In Burdett and Mortensen (1998), there is only one source of unemployment due to the infrequent arrival of job offers. However, in our model, there are two sources of labor market frictions. Not only must a worker apply for jobs, she must also have positive employment prospects. Equation (4) denotes the true unemployment rate $u$ and equation (5) an unemployment rate in which the only impediment to finding work is the frequency in which one applies to jobs, $\tilde{u}$. A comparison of the two rates reveals the relative quantitative importance of the two sources of frictions.

The exit rate from unemployment occurs after a $\gamma_{u}$ shock and on top of this, the worker must also have at least one potential job, which occurs with probability $\left(1-\Sigma_{0}(0)\right)$. The frictional rate is governed by only the primitive $\gamma_{u}$ which prevents a worker from matching with her current opportunities. The relative importance of the two frictions, by skill group, is reported in Table 4. The quantitative importance of a lack of opportunity is apparent, with this mechanism being responsible for approximately half of the unemployment rate.

\subsection{Frictional wage dispersion}

As has been discussed, in order to generate the level of frictional wage dispersion observed in the data, a typical search model requires an implausibly low or even negative flow benefit associated with unemployment. Our results, see Table 3, show that the implied replacement ratio, the ratio of the flow benefit to the mean wage in the economy needed to justify the observed wage distribution, is much higher under the baseline model. Across all skill groups, only the baseline model predicts a positive replacement 
ratio and depending on the skill, the other two specifications require large costs associated with unemployment. To put our numbers in some context, they are consistent with the macro labor literature (e.g., Shimer (2005) and Elsby and Michaels (2013)) as well as estimates from a field experiment which put the replacement ratio at 58\% (Mas and Pallais (2019)).

The replacement ratio in the Burdett and Mortensen (1998) model can be decomposed into the min-mean ratio and the search option,

$$
\underbrace{\frac{b}{\mathrm{E}[w]}}_{\text {Rep. ratio }}=\underbrace{\frac{w(0)}{\mathrm{E}[w]}}_{M m}+\underbrace{\int_{0}^{1} \frac{w^{\prime}(\tilde{F})}{\mathrm{E}[w]} \frac{\left(\lambda_{e}-\lambda_{u}\right)(1-\tilde{F})}{\delta+\mu+\lambda_{e}(1-\tilde{F})} d \tilde{F}}_{\text {Search }} .
$$

The flow value in our model is instead

$$
\begin{aligned}
\underbrace{\frac{b}{\mathrm{E}[w]}}_{\text {Rep. ratio }}=\underbrace{\frac{w(0)}{\mathrm{E}[w]}}_{M m}+\underbrace{\int_{0}^{1} \frac{w^{\prime}(\tilde{F})}{\mathrm{E}[w]} \frac{\gamma_{e}\left(+1-\Sigma_{e}(\tilde{F})\right)-\gamma_{u}\left(1-\Sigma_{u u}(\tilde{F})\right)}{\delta+\mu+\gamma_{e}\left(1-\Sigma_{e}(\tilde{F})\right)} d \tilde{F}}_{\text {Search }} \\
+\delta \underbrace{\left(\frac{\gamma_{u} \int_{0}^{1} \frac{w^{\prime}(\tilde{F})}{\mathrm{E}[w]} \frac{\Sigma_{u u}(\tilde{F})-\Sigma_{u}(\tilde{F})}{\delta+\mu+\gamma_{e}\left(1-\Sigma_{e}(\tilde{F})\right)} d \tilde{F}}{\left(\mu+\gamma_{u}\left(1-\Sigma_{u}(0)\right)\right)}\right)}_{\text {Insurance }} .
\end{aligned}
$$

The terms are intuitive; there are differences in the flow value and in the search option, captured by how often search occurs, the sampling distribution of wages and finally because, in our model, workers who separate from a job are in a different position as compared to the average unemployed. The second term, $\gamma_{i}\left(1-\Sigma_{i}(\tilde{F})\right)$, differs because the unemployed and employed do, on average, sample from different distributions, $\Sigma_{i}(\tilde{F})$, and at different rates, $\gamma_{i}$. Faberman et al. (2016) provided evidence that the employed on average sample from a better distribution. Similarly, the third term, $\Sigma_{u u}(\tilde{F})-\Sigma_{u}(\tilde{F})$, captures the effect that workers moving from employment to unemployment do, on average, have a higher number of prospects than the average unemployed, which generates the declining job finding rate with the duration of unemployment observed in the data. These effects are missed in the standard job ladder model. Table 5 provides a thorough decomposition of the replacement ratios for the different specifications and skill groups. A consistent finding across all skill groups is that only the baseline specification can accommodate the degree of frictional wage dispersion with a positive replacement ratio. An inspection of Table 5 reveals that while the insurance option helps, it is the reduction in the search option, which is quantitatively more important. While still negative, the better average prospects that the employed are exposed to significantly reduce the value of waiting in unemployment and, consequently, unemployed workers for the same value of $b$ are prepared to accept much lower wages. 
TABLE 5. Decomposition of the flow value of unemployment.

\begin{tabular}{lccccccrr}
\hline & $\begin{array}{c}\text { Replacement } \\
\text { Ratio }\end{array}$ & $\begin{array}{c}\text { Min-mean } \\
\text { Ratio }\end{array}$ & $\begin{array}{c}\text { Search } \\
\text { Option }\end{array}$ & $\begin{array}{c}\text { Insurance } \\
\text { Option }\end{array}$ & $\begin{array}{c}\text { Replacement } \\
\text { Ratio }\end{array}$ & $\begin{array}{c}\text { Min-mean } \\
\text { Ratio }\end{array}$ & $\begin{array}{r}\text { Search } \\
\text { Option }\end{array}$ & $\begin{array}{c}\text { Insurance } \\
\text { Option }\end{array}$ \\
\hline NDT & All & & & & High Skill & & & \\
NOJS & $-53.6 \%$ & $58.5 \%$ & $-112.1 \%$ & $0 \%$ & $-44.4 \%$ & $49.2 \%$ & $-93.6 \%$ & $0 \%$ \\
Baseline & $-290.2 \%$ & $54.4 \%$ & $-366.2 \%$ & $21.5 \%$ & $-714.1 \%$ & $46.2 \%$ & $-781.1 \%$ & $20.8 \%$ \\
& $35.0 \%$ & $59.5 \%$ & $-29.4 \%$ & $4.9 \%$ & $46.2 \%$ & $52.7 \%$ & $-8.6 \%$ & $2.2 \%$ \\
NDT & Medium Skill & & & & Low Skill & & & \\
NOJS & $-35.8 \%$ & $63.4 \%$ & $-99.2 \%$ & $0 \%$ & $-17.7 \%$ & $61.4 \%$ & $-79.1 \%$ & $0 \%$ \\
Baseline & $-200.0 \%$ & $60.5 \%$ & $-279.3 \%$ & $18.9 \%$ & $-46.4 \%$ & $62.3 \%$ & $-128.8 \%$ & $20.1 \%$ \\
\hline
\end{tabular}

Note: This table provides results from decomposing the replacement ratio into its three constituent parts derived in equations (11) and (12).

\subsection{Earnings loss}

Our baseline model and the two alternative specifications provide very different predictions regarding the average wage an unemployed worker receives in employment as a function of the duration of her unemployment spell. As is displayed in Figure 3, with no dynamic market thickness, an unemployed worker samples from the same distribution of wages, independent of the duration of their unemployment spell to date. However, because of selection into better jobs, the job ladder, this wage is lower than the mean wage among employed workers. Without OJS, there is no selection into better jobs for the employed. Thus, the average wage taken by an unemployed worker equals the average wage amongst employed workers. However, as a result of dynamic selection, a worker with a longer duration of unemployment will, on average, have fewer prospects, and thus samples from a distribution with a lower mean wage. This results in a decline in the average starting wage within the first couple of months. Our baseline model has both of these features, and thus generates both an average earnings loss, via selection in employment, and increasing losses with the duration of unemployment via dynamic selection.

Qualitatively, these two features: lower mean wages of new hires and declining wages with the duration of an unemployment spell mirror the data; see, for example, Addison and Portugal (1989). To assess whether our model matches the process quantitatively would require a thorough empirical examination beyond the scope of this paper. In particular, there is a large literature showing dispersion in earnings losses which vary with labor market features that are not specifically modeled in this paper. ${ }^{23}$

\subsection{Search process}

To demonstrate that our model replicates workers' search behavior in a realistic manner, we compare the underlying theoretical mechanism with direct evidence on work-

\footnotetext{
${ }^{23}$ Empirical studies have shown earnings losses vary by: industry/occupation Stevens (1997); local labor market conditions Carrington (1993); and Couch and Placzek (2010).
} 
All
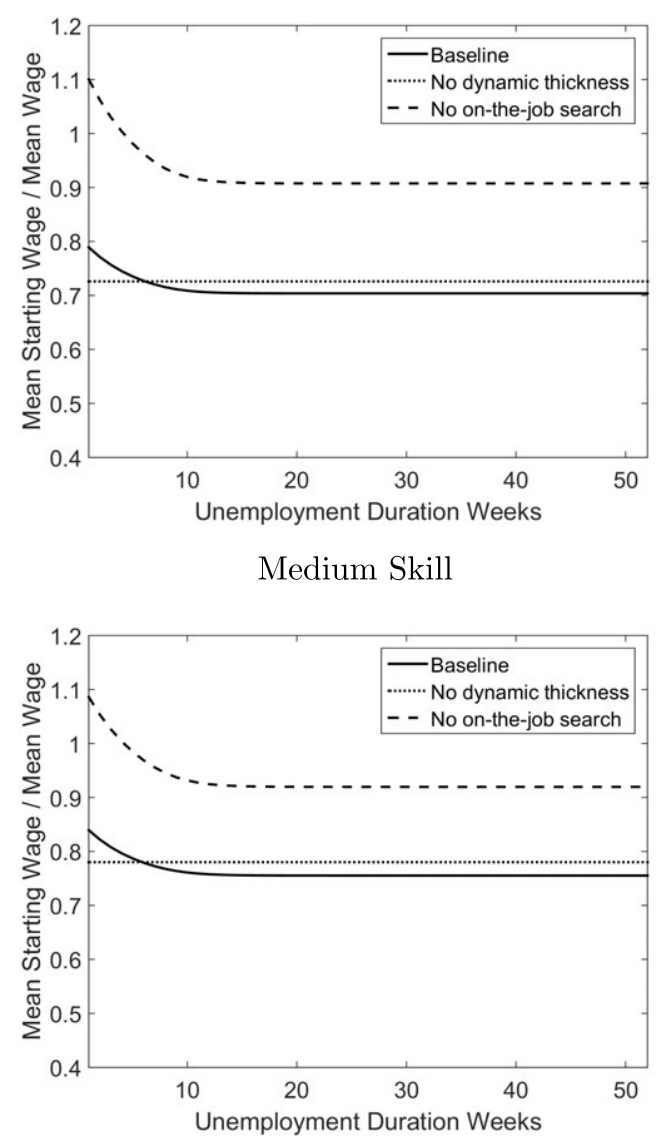

High Skill
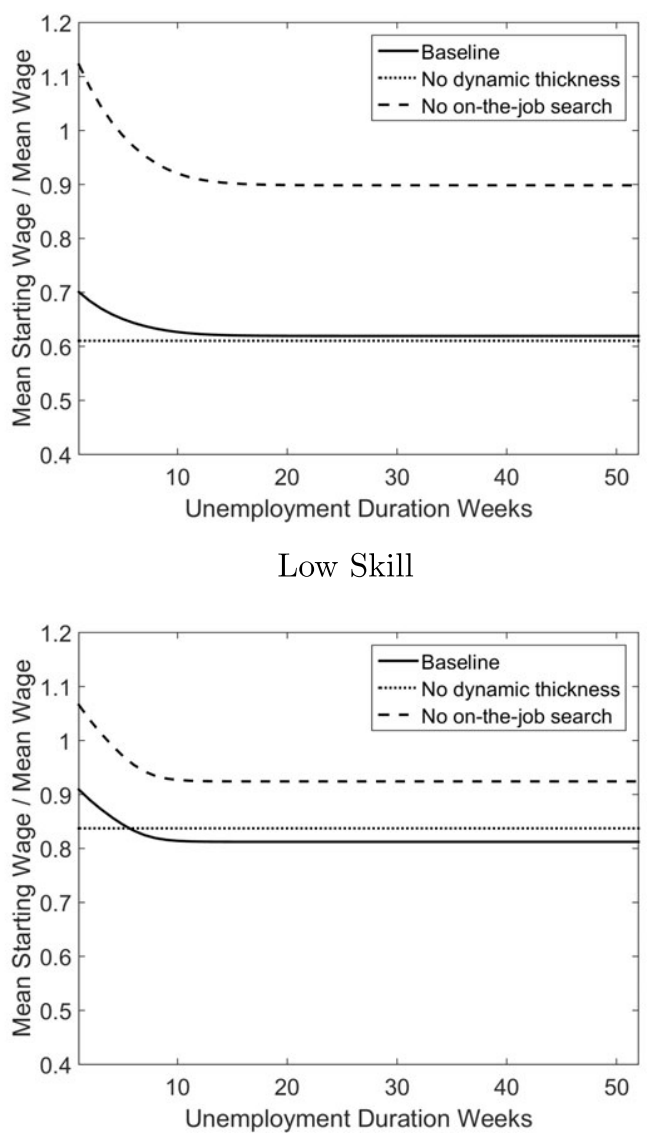

Figure 3. Wage loss after job loss. Note: The figure shows the mean wage an unemployed agent receives by the duration of their unemployment spell. This wage is reported relative to the mean wage in the labor market as a whole.

ers' search behavior. For this exercise, we rely on two data sources not used in our estimation. They constitute a supplement to the Survey of Consumer Expectations (SCE) provided by the New York Fed from 2013 and 2014 and has recently also been used by Faberman et al. (2016). The survey is a repeated cross-section, nationally representative and has approximately 1200 individuals per year. In addition, we use the Survey of Unemployed Workers in New Jersey. The data and their construction are detailed in Krueger and Mueller (2011). 6025 unemployed workers in the New Jersey area are surveyed at a weekly frequency for up to 24 weeks. A feature of the data is that it asks workers about the job offers they receive (not necessarily take) and their contemporaneous reservation wage. ${ }^{24}$ Results from both data sets are weighted by the weights provided and described in Faberman et al. (2016) and Krueger and Mueller (2016), respectively.

\footnotetext{
${ }^{24}$ The data for this analysis are available for public download at http://opr.princeton.edu/archive/njui/.
} 
(a) Offer Distribution

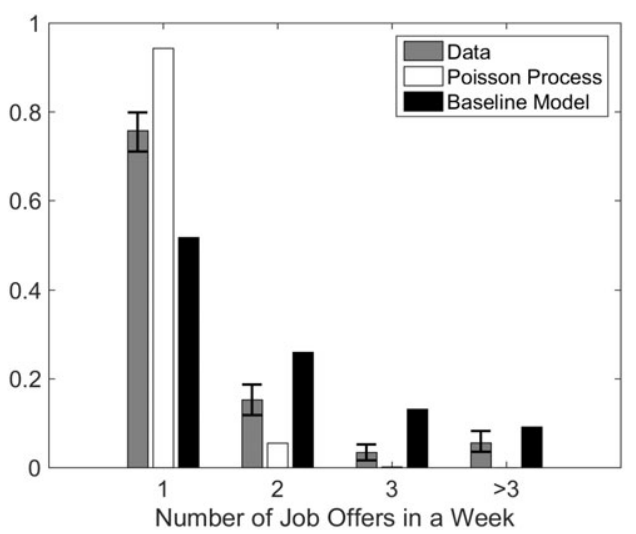

(b) Log Ratio

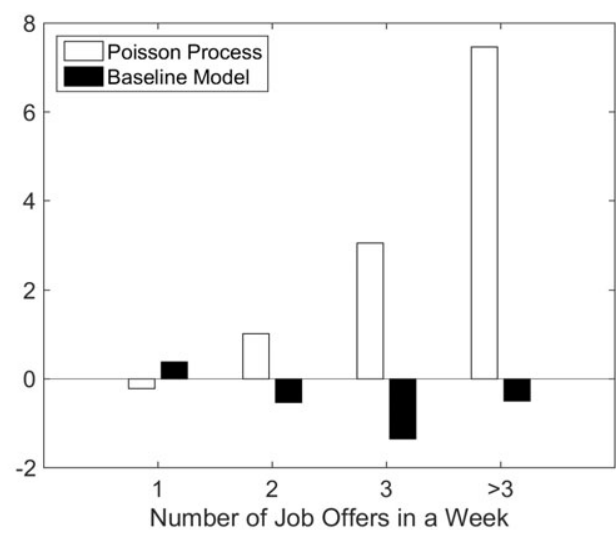

Figure 4. Number of job offers. Note: The data come from the survey of unemployed job seekers in New Jersey. We restrict our attention to male workers between the age of 25 and 45 . Panel (a) shows the distribution of reported job offers received in a week, conditional on receiving at least one offer: as observed empirically (weighted by sampling weights); implied by a Poisson process, and as implied by the baseline model. Our interpretation of offers in the model is the distribution of opportunities held by the unemployed, $p_{u}(j)$. Panel (b) is the log ratio of the implied distributions with the data.

First, we exploit data from the Survey of Unemployed Workers in New Jersey. In Figure 4, we present the number of offers received by unemployed workers in a month and compare this to what is predicted by our model and a memoryless Poisson process. The memoryless Poisson process is computed, given the proportion of people in the data with no offers. Our baseline model is a representation of the distribution $p_{u}(j)$, the solution of the flow equations (15) and (16). Since the data cover a different time period and only focus on New Jersey, there is no reason to assume that the model will fit the data well. However, what is clear from panel (b) is that a memoryless Poisson process cannot generate the number of people with large numbers of offers that is observed in the data-a feature that our baseline model replicates.

Turning to the supplement of the Survey of Consumer Expectations (SCE), it is worth noting that the statistics presented here are merely to demonstrate that the underlying search process reported in the survey is quite different from what is assumed in a standard search model and bears some resemblance to the mechanism in our model. Any further inference is difficult to make since although representative, the sample has a fairly small cross-section, meaning that inference about the unemployed is based on 61 (26) individuals (males). Table 6 shows by employment status, over a 4-week period, the mean number of applications, the proportion of those making at least one application, and the mean number of contacts received. We present these from the model and the data, and we further distinguish between the unemployed and long-term unemployed in our model. The unemployed do, on average, send out more applications and more frequently engage in active search as compared to their employed counter- 
TABLE 6. Mean job prospects by employment status.

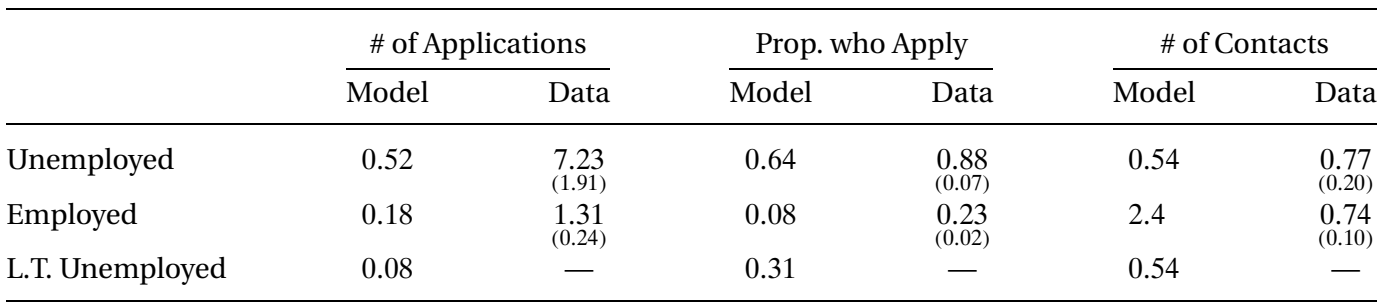

Note: The data are taken from the Survey of Consumer Expectations and the attention is restricted to male workers. Applications are calculated based on the question "How many potential employers, if any, did you apply to for employment within the LAST 4 WEEKS? Please include all applications made in person, online, or through other direct methods. Do not include inquiries that did not lead to a job application." Similarly, the number of contacts are computed based on the question "In the LAST 4 WEEKS, how many potential employers contacted you about a job opening? Please include all contacts, even those that were not solicited by you." All moments are computed based on appropriate sampling weights. Long-term unemployed is defined as having reached the ergodic distribution of prospects, in practice this occurs in under 3 months. In the model, the proportion who apply is computed as the proportion who actively apply and have positive prospects. The number of contacts are the total number of prospects applied to. Reported in the parentheses are standard errors.

parts. A fact that our model successfully reconciles. ${ }^{25}$ The number of contacts received in a month, on the other hand, is similar across the two groups. However, since we have relatively few unemployed in the sample, it is hard to establish this clearly. What is certain is that, as implied by a standard job ladder model, the unemployed do not receive an order of magnitude larger contacts than their employed counterparts. Finally, we have included the model's predictions for the long-term unemployed to further inform the reader. However, with so few unemployed in the data, the same moments in the data are uninformative.

\section{Conclusion}

This paper sets up a model, which extends the standard job ladder model to incorporate thin markets. The model is solved analytically and estimated on U.S. survey data. The estimated model delivers declining job finding rates by the duration of unemployment as observed in the data. Further, the flow value associated with unemployment required to match the wage distribution does not need to be large and negative. Our estimates of the replacement ratio, in the order of a quarter to a half of the workers' average wage, are consistent with the numbers used in the macro labor literature. On the other hand, the estimation of the Burdett and Mortensen (1998) model and a model without on-the-job search requires large and negative replacement ratios. Additionally, to generate a wage penalty associated with the duration of an unemployment spell, the standard job ladder model requires decreasing general human capital in unemployment. Our model generates this via the stochastic process for employment prospects. This has implications for the persistence in earnings losses following job displacement. Whether this mechanism can generate a sufficient persistence in earnings remains an open question and could prove fruitful for future research.

\footnotetext{
${ }^{25}$ The number of applications sent out in the data is significantly higher than what is predicted by the model. This difference could be driven by differences in applications and our model definition of a suitable opportunity. The data is also sensitive to a few people are observed sending out hundreds of applications.
} 


\section{References}

Addison, J. and P. Portugal (1989), "Job displacement, relative wage changes, and duration of unemployment.” Journal of Labor Economics, 7, 281-302. [1423]

Albrecht, J., P. Gautier, and S. Vroman (2006), "Equilibrium directed search with multiple applications.” The Review of Economic Studies, 73, 869-891. [1402]

Alvarez, F., K. Borovickova, and R. Shimer (2019), "Decomposing duration dependence in a stopping time model.” Report. [1420]

Alvarez, F. and R. Shimer (2011), "Search and rest unemployment." Econometrica, 79, 75-122. [1400]

Barlevy, G. (2008), "Identification of search models using record statistics.” The Review of Economic Studies, 75, 29-64. [1409]

Belot, M., P. Kircher, and P. Muller (2019), "Providing advice to jobseekers at low cost: An experimental study on online advice.” The Review of Economic Studies, 86, 1411-1447. [1401]

Bontemps, C., J.-M. Robin, and G. V. den Berg (2000), "Equilibrium search with continuous productivity dispersion: Theory and nonparametric estimation." International Economic Review, 41, 305-358. [1410]

Bound, J., C. Brown, and N. Mathiowetz (2001), "Measurement error in survey data." Handbook of Econometrics, 5, 3705-3843. [1413]

Bradley, J. and A. Gottfries (2021), "Supplement to 'A job ladder model with stochastic employment opportunities'” Quantitative Economics Supplemental Material, 12, https: //doi.org/10.3982/QE1394. [1405, 1413]

Bradley, J., F. Postel-Vinay, and H. Turon (2017), "Public sector wage policy and labor market equilibrium: A structural model." Journal of the European Economic Association, 15, 1214-1257. [1399]

Burdett, K., C. Carrillo-Tudela, and M. Coles (2020), “The cost of job loss.” The Review of Economic Studies, 87, 1757-1798. [1402]

Burdett, K. and M. Coles (2003), “Equilibrium wage-tenure contracts.” Econometrica, 71, 1377-1404. [1412]

Burdett, K. and M. Coles (2010), "Wage/tenure contracts with heterogeneous firms." Journal of Economic Theory, 145, 1408-1435. [1412]

Burdett, K. and K. Judd (1983), “Equilibrium price dispersion.” Econometrica, 51, 955969. [1408, 1410, 1412]

Burdett, K. and D. T. Mortensen (1998), "Wage differentials, employer size, and unemployment.” International Economic Review, 257-273. [1400, 1401, 1408, 1409, 1410, 1411, 1412, 1421, 1422, 1426] 
Cahuc, P., F. Postel-Vinay, and J.-M. Robin (2006), "Wage bargaining with on-the-job search: Theory and evidence.” Econometrica, 74, 323-364. [1403]

Carillo-Tudela, C. and E. Smith (2016), “Search capital.” Review of Economic Dynamics, 23, 191-211. [1405]

Carrillo-Tudela, C. (2009), "An equilibrium search model when firms observe workers' employment status.” International Economic Review, 50, 485-506. [1403]

Carrillo-Tudela, C. and L. Visschers (2013), "Unemployment and endogenous reallocation over the business cycle.” Report. [1400]

Carrington, W. J. (1993), "Wage losses for displaced workers: Is it really the firm that matters?” Journal of Human Resources, 28, 435-462. [1423]

Coles, M. G. and E. Smith (1998), “Marketplaces and matching.” International Economic Review, 239-254. [1400, 1410]

Couch, K. A. and D. W. Placzek (2010), "Earnings losses of displaced workers revisited.” American Economic Review, 100, 572-589. [1423]

Elliott, M. (2014), “Heterogeneities and the fragility of labor markets.” Report. [1403]

Elsby, M. W. L. and R. Michaels (2013), "Marginal jobs, heterogeneous firms, and unemployment flows.” American Economic Journal: Macroeconomics, 5, 1-48. [1402, 1422]

Faberman, R. J., A. Mueller, A. Şahin, and G. Topa (2016), "Job search behavior among the employed and non-employed.” Report. [1401, 1403, 1404, 1422, 1424]

Fujita, S. and G. Moscarini (2017), "Recall and unemployment.” American Economic Review, 107, 3875-3916. [1405]

Gautier, P. and C. Holzner (2017), "Simultaneous search and efficiency of entry and search intensity.” American Economic Journal: Microeconomics, 9, 245-282. [1403]

Gottfries, A. (2019), "Bargaining with renegotiation in models with on-the-job search." Report. [1412]

Hagedorn, M. and I. Manovskii (2008), "The cyclical behavior of equilibrium unemployment and vacancies revisited.” The American Economic Review, 98, 1692-1706. [1402]

Hornstein, A., P. Krusell, and G. Violante (2011), "Frictional wage dispersion in search models: A quantitative assessment.” American Economic Review, 101, 2873-2898. [1401, 1403]

Jarosch, G. (2015), “Searching for job security and the consequences of job loss.” Report. [1402]

Kircher, P. (2009), “Efficiency of simultaneous search.” Journal of Political Economy, 117, 861-913. [1403]

Kircher, P. and M. Galenianos (2009), "Directed search with multiple job applications.” Journal of Economic Theory, 114, 445-471. [1402, 1403] 
Krolikowski, P. (2017), "Job ladders and earnings of displaced workers.” American Economic Journal: Macroeconomics, 9, 1-31. [1402]

Krueger, A. B. and A. Mueller (2011), "Job search, emotional well-being and job finding in a period of mass unemployment: Evidence from high-frequency longitudinal data." Brookings Papers on Economic Activity, 42, 1-81. [1424]

Krueger, A. B. and A. I. Mueller (2012), "The lot of the unemployed: A time use perspective." Journal of the European Economic Association, 10, 765-794. [1404]

Krueger, A. B. and A. I. Mueller (2016), "A contribution to the empirics of reservation wages.” American Economic Journal: Economic Policy, 8, 142-179. [1402, 1403, 1424]

Lucas, R. E. and E. C. Prescott (1974), “Equilibrium search and unemployment.” Journal of Economic theory, 7, 188-209. [1400]

Mas, A. and A. Pallais (2019), "Labor supply and the value of non-work time: Experimental estimates from the field.” American Economic Review: Insights, 1, 111-126. [1422]

McCall, J. J. (1970), “Economics of information and job search.” Quarterly Journal of Economics, 84, 113-126. [1400]

Meghir, C., R. Narita, and J.-M. Robin (2015), "Wages and informality in developing countries.” American Economic Review, 105, 1509-1546. [1399]

Mortensen, D. T. and C. A. Pissarides (1994), "Job creation and job destruction in the theory of unemployment." The Review of Economic Studies, 61, 397-415. [1405]

Ortego-Marti, V. (2016), “Unemployment history and frictional wage dispersion.” Journal of Monetary Economics, 78, 5-22. [1403]

Papp, T. K. (2013), "Frictional wage dispersion with Bertrand competition: An assessment.” Review of Economic Dynamics, 16, 540-552. [1403]

Postel-Vinay, F. and J.-M. Robin (2002), "Equilibrium wage dispersion with worker and employer heterogeneity.” Econometrica, 70, 2295-2350. [1403, 1412]

Shimer, R. (2005), "The cyclical behavior of equilibrium unemployment and vacancies.” American Economic Review, 95, 25-49. [1402, 1422]

Shimer, R. (2007), “Mismatch.” American Economic Review, 97, 1074-1101. [1400]

Sleet, C. and H. Yazici (2017), “Taxation, redistribution and frictional labor supply.” Report. [1399]

Stevens, A. H. (1997), "Persistent effects of job displacement: The importance of multiple job losses.” Journal of Labor Economics, 15, 165-188. [1423]

Stevens, M. (2004), “Wage-tenure contracts in a frictional labour market: Firms' strategies for recruitment and retention." The Review of Economic Studies, 71, 535-551. [1412]

Stigler, G. J. (1962), “Information in the labor market.” Journal of political economy, 70, 94-105. [1400] 
Wolthoff, R. (2014), "It's about time: Implications of the period length in an equilibrium search model.” International Economic Review, 55, 839-867. [1403]

Wolthoff, R. (2017), “Applications and interviews: Firms' recruiting decisions in a frictional labour market.” The Review of Economic Studies, 85, 1314-1351. [1403]

Co-editor Christopher Taber handled this manuscript.

Manuscript received 25 July, 2019; final version accepted 22 March, 2021; available online 29 April, 2021. 